\title{
A informação na consulta presencial em Medicina Geral e Familiar: classificações segundo a ICPC-2 e anotações livres para a memória futura no SOAP
}

Information available in general practice/family medicine consultations: coding and annotations for future use

\section{La información en la consulta médica presencial en Medicina General y Familiar: clasificaciones según ICPC-2 y apuntes libres para la memoria futura en el SOAP}

Luiz Miguel Santiago. Administração Regional de Saúde do Centro, IP (ARSC). Coimbra, Portugal. Universidade da Beira Interior. Covilhã, Portugal. Imsantiago @netcabo.pt. (autor correspondente)

Rosa Carvalho. USF Topázio, ARSC do Centro. Coimbra, Portugal. rosa.moura.carvalho@gmail.com

Philippe Botas. USF Topázio, ARSC do Centro. Coimbra, Portugal. phbotas@gmail.com

Paula Miranda. USF Topázio, ARSC do Centro. Coimbra, Portugal. paula.ricardo.miranda@gmail.com

Catarina Matias. USF Topázio, ARSC do Centro. Coimbra, Portugal. catarinaismatias@gmail.com

Ana Rita Simões. USF Topázio, ARSC do Centro. Coimbra, Portugal. anaritasims@gmail.com

Carolina Pereira. USF Topázio, ARSC do Centro. Coimbra, Portugal. carolinaduartepereira @ gmail.com

Maria Glória Neto. USF Topázio, ARSC do Centro. Coimbra, Portugal. Iola.neto@gmail.com

\section{Resumo}

Objetivos: assumindo a obrigatoriedade de classificação ICPC-2 em cada consulta, conhecer a informação, em consultas passadas pelo método de Weed-SOAP segundo o gênero e idade de quem consulta o médico (consulente ou paciente), caracterizando o nível de registro pelo método SOAP em Subjetivo (S) - classificação e anotações - em Objetivo $(O)$ anotações sobre o estado do paciente, em Avaliação $(A)$ da classificação e em Plano $(P)$ da classificação e anotações. Métodos: estudo observacional, transversal em outubro de 2012, em amostra aleatorizada das consultas presenciais de dois médicos orientadores de internato de especialidade, em três meses sorteados do 1 은 semestre de 2012, e em quatro dias sorteados em cada mês, em amostra representativa com intervalo de confiança de $95 \%$ e margem de erro de $6 \%$. Utilizou-se estatística descritiva e inferencial. Resultados: amostra de 318 consultas, $n=149$ $(46,9 \%)$ no gênero masculino, $n=61(19,2 \%)$ no grupo etário <18 anos e $n=194(61,0 \%)$ no $\geq 18$ e <65 anos, ns por grupos etários e gênero. Em S, há classificação em $98,7 \%$ e anotação em $47,2 \%$ das consultas; Em O, verificamos "As anotações demonstram o estado do paciente" em 66,0\% e "As anotações são explícitas e entendíveis" em 79,9\%; em A, $97,8 \%$ das consultas têm classificação; Em P, há classificação em 96,5\% e anotações explicando o plano em $23,0 \%$ das consultas. Distribuição sem significado por grupo etário para as variáveis estudadas. É mais frequente haver no gênero feminino em S "As anotações são explícitas e entendíveis" e em P "Há classificação de procedimentos". Conclusão: há campo para mais completa coleta da informação na consulta, permitindo, assim, melhor conhecimento de cada consulta e caso para o futuro.
Palavras-chave:

Referência e Consulta

Classificação Internacional

de Atenção Primária

Registros Médicos
Como citar: Santiago LM, Carvalho R, Botas P, Miranda P, Matias C, Simões AR, Pereira C, Glória Neto M. A informação na consulta presencial em Medicina Geral e Familiar: classificações segundo a ICPC-2 e anotações livres para a memória futura no SOAP. Rev Bras Med Fam Comunidade. 2015;10(36)1-8. http://dx.doi.org/10.5712/rbmfc10(36)840
Fonte de financiamento: declaram não haver. Parecer CEP: 6/2014 (ARSC) aprovado em 27/02/2014.

Conflito de interesses: declaram não haver. Procedência e revisão por pares: revisado por pares. Recebido em: 20/10/2013. Aprovado em: 04/08/2015. 


\section{Abstract}

Objectives: assuming the mandatoriness of ICPC2 classification in every consultation, the objective of this study was to ascertain the frequency of this type of classification in past consultations. This analysis was performed using the Weed-SOAP method, where Subjective (S) is measured by classifications and annotations, Objective (O) by annotations, Avaliation $(A)$ by classification, and Plan (P) by classification and annotations. Methods: a cross-sectional observational study was performed in October 2012, with a 95\% confidence interval and $6 \%$ error margin, on a representative random sample of consultations conducted on 4 random days of 3 random months of the first semester of 2012, and data were analyzed using descriptive and inferential statistics. Results: among the sample of 318 consultations, $149(46.9 \%)$ were with male patients and 61 (19.2\%) were aged under 18 years, while $194(61.0 \%)$ were aged 18 or above but under 65 years, after sorting by age groups and gender. In terms of S, $98.7 \%$ of consultations had an ICPC-2 classification and $47.2 \%$ had an annotation; in terms of $0,66.0 \%$ had an annotation demonstrating the state of the patient and $79.9 \%$ were explicit; in terms of $A, 97.8 \%$ had a classification; and in terms of $P, 96.5 \%$ had a classification and $23.0 \%$ had an annotation explaining the plan. There was no statistically significant difference by age group for the studied variables. However, for gender, women had more "Clear and explicit" annotations in S, and "Classification of plan" instances in P. Conclusion: more complete recall of information in consultations is required in order to gain better knowledge about individual consultations and patients for future use.

\section{Resumen}

Objetivos: asumiendo la obligatoriedad de clasificar con la ICPC-2 en cada consulta, conocer la información, en consultas tras-efectuadas pelo método Weed-SOAP según el género y edad de quien consulta al médico (los pacientes), caracterizando el nivel de registro por el método SOAP en Subjetivo (S) - clasificación y anotaciones - en Objetivo (O) apuntes sobre el estado del paciente, en Evaluación $(A)$ de la clasificación y en Pleno $(P)$ de la clasificación y apuntes. Métodos: estudio observacional, transversal en octubre del 2012, en muestra aleatoria de las consultas presenciales de dos médicos tutores de residencia de la especialidad, por tres meses sorteados del $1 \%$ semestre del 2012 y en cuatro días sorteados en cada mes, en muestra representativa con intervalo de seguridad del $95 \%$ y margen de error del $6 \%$. Se utilizó estadística descriptiva e inferencial. Resultados: muestra de 318 consultas, $n=149(46,9 \%)$ en el género masculino, $n=61$ $(19,2 \%)$ en el grupo de edad $<18$ años y $n=194(61,0 \%)$ en el $\geq 18$ y $<65$ años, ns por grupos de edad y género. En $S$, hay clasificación en el $98,7 \%$ y apuntes en el $47,2 \%$ de las consultas; En O, verificamos "Los apuntes demuestran el estado del paciente" en $66,0 \%$ y "Los apuntes son explícitos y comprensibles" en el 79,9\%; en A, el $97,8 \%$ de las consultas tienen clasificación; En P, hay clasificación en el 96,5\% y apuntes explicando el plan en el 23,0\% de las consultas. Distribución sin significado por grupo de edad para las variables estudiadas. Es más frecuente haber en el género femenino en $S$ "Los apuntes son explícitos y comprensibles" y en P "Hay clasificación de procedimientos". Conclusión: hay campo para un recogido de informaciones más completo, permitiendo de esta manera, mejor conocimiento de cada consulta en el futuro.

\section{Keywords:}

Referral and Consultation International Classification of Primary Care Medical Records

Palabras clave: Remisión y Consulta Clasificación Internacional de la Atención Primaria Registros Médicos

\section{Introdução}

A informação do ocorrido em um contato entre médico e paciente deve ser guardada para memória futura quer na forma da escrita do ocorrido - a que chamaremos de anotações -, quer na forma de alocação de conhecimento alfa-numérico - a que chamaremos de classificação - quer numa simbiose entre as duas.

Em Portugal, segundo o Código Deontológico da ordem dos Médicos, ${ }^{1}$ compete ao médico, artigo $68^{\circ}$ ponto 2, a obrigação de segredo e segundo o artigo 69 ponto 5 "A guarda, o arquivo e a superintendência nos processos clínicos dos doentes...". Já quanto ao âmbito do segredo profissional, o Código refere no artigo 77을 ponto 2 "A ficha clínica do doente, que constitui a memória escrita do Médico, pertence a este e não àquele...". Segundo a Lei no 46/2007 de 24 de Agosto, " "A comunicação de dados de saúde é feita por intermédio de médico..." o que implica a sua existência e devido exercício de zelo na sua elaboração, atualização e guarda e a Lei no 12/2005 de 26 de Janeiro ${ }^{3}$ com o título "Informação genética pessoal e informação de saúde", no seu artigo 2ำ, diz que "Para os efeitos desta lei, a informação de saúde abrange todo o tipo de informação directa ou indirectamente ligada à saúde...", referindo o artigo 3 o (Propriedade da informação de saúde) que "1 - A informação de saúde, incluindo os dados clínicos registados, resultados de análises e outros exames subsidiários, intervenções e diagnósticos, é propriedade da pessoa...".

No ambiente da consulta de Medicina Geral e Familiar (MGF) ${ }^{4-9}$ o sistema de coleção de informação é realizado de uma forma sistemática segundo a organização de Subjetivo (S) - as queixas, sinais ou sintomas 
do paciente ou as suas necessidades para o momento, ou seja, o lado psicossocial da consulta que pode ser também bio, Objetivo $(O)$, em que se realiza o assentamento do colhido na consulta quer física quer psiquicamente, a Avaliação (A), o capítulo do diagnóstico que o médico realiza e o Plano ou Procedimento $(P)$, conjunto de informação relativa ao que se realizou e se pede ou se solicita que o paciente faça.

Os registros na consulta poderiam ser realizados apenas com anotações. No entanto, a uniformização da informação levou à criação de sistemas de classificação com atribuição de códigos alfa-numéricos a entidades nosológicas (CID) reconhecendo, desde 2003, a OMS, a ICPC-2 como a melhor forma de codificar em MGF. ${ }^{10}$ Esta classificação permite não só codificar a doença que o médico diagnostica como também o motivo para a consulta e o conjunto de pedidos, de informação e de atos realizados para um paciente num contato médico-paciente. No capítulo do diagnóstico (A) ICPC-2 e CID 10 são emparelháveis.

Existe debate quanto à profundidade e à extensão em que a ICPC-2 deve ser utilizada em MGF, ${ }^{11-14}$ por ser o próprio a realizar a classificação, ao contrário do ambiente hospitalar em que há uma comissão de classificação e quanto à classificação em $S$ e em $P$ pela eventual alteração que possa colocar no processo da consulta por o médico passar algum tempo a olhar para a tela de um computador, apesar de não se perceber qual a diferença entre olhar para a tela e olhar para uma folha de papel onde se escreve com caligrafia por vezes ilegível.

A consulta em MGF necessita boa perceção e eventual classificação, da agenda de problemas do doente, implica uma boa semiologia, exige correta classificação dos problemas diagnosticados e obriga ao conhecimento do plano desenhado, bem como dos procedimentos realizados num paciente.

Os campos S, A e P podem ser classificados para memória futura sobre o que aconteceu numa consulta. Este conhecimento, bem como as anotações porventura esclarecedoras da classificação, até por haver situações em que a classificação não cobre todas as queixas, é importante que seja reconhecido pelo médico, bem como por outros médicos que venham a ter contato posterior com o consulente. Mesmo o paciente, ao ter acesso aos seus dados, pode e deve perceber o que foi anotado pelo médico. De fato, quando questões legais possam surgir, só a boa informação registada poderá dirimir problemas ou dúvidas.

A este propósito, o Conselho Nacional de Ética Para as Ciências da Vida ${ }^{15}$ no seu "Parecer Sobre Informação de Saúde e Registros Informáticos de Saúde”, refere:

Que os dados de saúde, enquanto dados sensíveis referentes à intimidade da pessoa, são reservados e sujeitos a dever de confidencialidade" e que "em qualquer altura do seu relacionamento com as instituições de saúde ou com profissionais de saúde qualquer cidadão tenha direito, podendo fazê-lo por escrito, a que determinado dado clínico relativo a si próprio (desde que imposições legais baseadas em razões de Saúde Pública o não impeçam) seja considerado absolutamente reservado e, consequentemente, vedado a outros acessos para além do acesso pelo profissional a quem foi voluntariamente transmitido. Para tanto, o profissional ou a instituição de saúde deverá, no momento do registro, ou logo que tal se torne possível, dar conhecimento à pessoa desse direito.

Dada a presente organização de recolha de informação na consulta, é necessário perceber como tal recolha deve ser feita para se perceber do que se tratou numa consulta. A classificação pode ajudar a estudos científicos, pois padroniza. Já a anotação é importante, para se poder melhor perceber a agenda de problemas, ter a descrição do estado do consulente e perceber-se o que foi delineado e/ou acordado com o consulente. 4,6,10 
Numa unidade de formação em pré e pós graduado em Medicina Geral e Familiar, na qual, por princípio, todos os campos do método Weed-SOAP devem ser classificados, surge a necessidade de realizar trabalho que permita saber a frequência, num ambiente de consultas efetuadas no passado e segundo o gênero dos pacientes e a sua idade da informação colocada no prontuário de registro clínico.

\section{Objetivos}

Conhecer os registros em S através da classificação e/ou da explicitação das anotações;

Conhecer as anotações em O percebendo se estas demonstram o estado do consulente e se são explícitas e entendíveis;

Conhecer a informação em A pela classificação;

Conhecer os registros em $\mathrm{P}$ para saber se há classificação de procedimentos e anotações que explicitem o plano.

\section{Métodos}

Estudo observacional, transversal analítico em amostra aleatorizada das consultas presenciais de dois médicos orientadores de internato de especialidade e de seus seis internos de especialidade em MGF. Por consulta presencial entende-se aquela em que o paciente esteve com o médico em consultório e, segundo o sistema português de taxas moderadoras, como tal faturada.

Dos primeiros seis meses de 2012, por sorteio sem reposição, foram selecionados três meses para estudo - fevereiro, abril e junho. Para cada mês, conhecido o número de consultas realizadas nesse mês pelo conjunto dos médicos em estudo, percebeu-se o número médio de consultas por dia. Foi calculado o tamanho da amostra de consultas a estudar para população finita, com um intervalo de confiança de 95\% e uma margem de erro de $6 \%$, como sendo de 332 consultas. Foi então conseguido o número de dias a estudar para representação de tamanho amostral, tendo sido, por cada mês, sorteados sem reposição os quatro dias a estudar. No Quadro 1, são mostrados os valores que acima são referidos. O número de consultas e a sua elegibilidade em função dos critérios acima foram retirados do Sistema de Informação das Unidades de Saúde (SINUS).

A análise dos dados, sigilosos e pertencentes aos pacientes e da guarda dos médicos, foi realizada em outubro de 2012, sempre pelos mesmos observadores, que registraram em impresso próprio. A folha de recolha de dados não continha campos relativos nem ao dia nem ao médico cujo trabalho era estudado. Foram destruídos os papéis de sorteio e não houve, nunca, qualquer identificação dos consulentes cujas consultas foram estudadas. Foram considerados os seguintes grupos etários: <18 anos; $\geq 18$ e <65 anos e $\geq 65$ anos. Foi obtido parecer positivo da Comissão de Ética da ARSC, no 6/2014, 27/02/2014.

Quadro 1. Explicitação do cálculo do tamanho da amostra de consultas a estudar.

\begin{tabular}{|lc|}
\hline Número de consultas presenciais & 2383 \\
Número efetivo de dias de consulta & 59 \\
Tamanho da amostra de consultas presenciais a estudar & 332 \\
Número médio de consultas presenciais por dia & 22 \\
Número de dias a estudar & 12 \\
Número de dias por mês & 4 \\
\hline
\end{tabular}


Foram estudadas a existência de classificação ICPC2 em S, A e P e a existência de anotações clínicas em S, O, A e P.

Foi utilizada estatística descritiva e não paramétrica pelo teste do $X^{2}$ e de Kruskal-wallis, definindo-se diferença com significado para valor de $p<0,05$.

\section{Resultados}

Segundo o Quadro 2, foi estudada uma amostra de 318 consultas, sendo $n=149(46,9 \%)$ no gênero masculino, $n=61(19,2 \%)$ ao grupo etário menor de 18 anos, $n=194(61,0 \%)$ ao grupo etário $\geq 18$ e $<65$ anos e n=63 (19,8\%) ao grupo etário $\geq 65$ anos, não havendo diferença significativa na distribuição de grupos etários por gênero.

No Quadro 3, mostramos a distribuição das variáveis medidas no global, sendo de destacar a elevada proporção com classificação realizada e as baixas frequências de anotações explicando códigos.

No Quadro 4, mostramos os resultados encontrados em função do gênero e do grupo etário, bem como a diferença estatística. Apenas se verificam diferenças significativas entre homem e mulher quanto a "As anotações são explícitas e entendíveis" em O e "Há classificação de procedimentos" em P que são mais frequentes no sexo feminino.

\section{Discussão}

Não sendo frequente em Portugal a necessidade de, em Tribunal ou outra instância, um médico ter de provar o que de fato realizou numa consulta, é, no entanto, frequente um médico verificar falta de informação suficiente para entender o que no passado ocorreu com um paciente seu. De fato, a memória do que aconteceu numa consulta é, por via da importância relativa atribuída a ir a uma consulta, mais

Quadro 2. Distribuição da amostra por gênero/grupo etário.

\begin{tabular}{|cccc|}
\hline & $\begin{array}{c}\text { Masculino(*) } \\
\mathbf{n}(\%)\end{array}$ & $\begin{array}{c}\text { Feminino(*) } \\
\mathbf{n}(\%)\end{array}$ & $\begin{array}{c}\text { Total } \\
\mathbf{n}(\%)\end{array}$ \\
\hline$<18$ anos & $31(20,8)$ & $30(17,8)$ & $61(19,2)$ \\
$\geq 18 e<65$ anos & $87(58,4)$ & $107(63,3)$ & $194(61,0)$ \\
$\geq 65$ anos & $31(20,8)$ & $32(18,9)$ & $63(19,8)$ \\
Total & $149(100)$ & $169(100)$ & $318(100)$ \\
\hline
\end{tabular}

$\left(^{\star}\right) p=0,868$.

Quadro 3. As variáveis medidas para a constatação do ambiente de percepção da informação na consulta.

\begin{tabular}{|c|c|c|}
\hline Capítulo & Variável & n (\%) \\
\hline \multirow{2}{*}{$\mathrm{S}$} & Classificação & $314(98,7)$ \\
\hline & A anotação explica classificação & $150(47,2)$ \\
\hline \multirow{2}{*}{$\mathrm{O}$} & As anotações demonstram o estado do consulente & $210(66,0)$ \\
\hline & As anotações são explícitas e entendíveis & $254(79,9)$ \\
\hline A & Há classificação(ões) que permita(m) pensar em diagnóstico & $311(97,8)$ \\
\hline \multirow{2}{*}{$\mathrm{P}$} & Há classificação de procedimentos/plano & $307(96,5)$ \\
\hline & Há anotações que explicitem o plano & $73(23,0)$ \\
\hline
\end{tabular}


Quadro 4. Distribuição das variáveis em função do gênero e do grupo etário.

\begin{tabular}{|c|c|c|c|c|c|c|c|c|}
\hline & \multicolumn{3}{|c|}{ Gênero } & \multicolumn{3}{|c|}{ Grupo etário } & \multirow[b]{2}{*}{ p } \\
\hline & & $\begin{array}{c}0^{\pi} \\
\text { n (\%) }\end{array}$ & $\begin{array}{c}\stackrel{9}{0} \\
\text { n }(\%)\end{array}$ & $\mathbf{p}$ & $\begin{array}{c}1 \\
\text { n (\%) }\end{array}$ & $\begin{array}{c}2 \\
\mathrm{n}(\%)\end{array}$ & $\begin{array}{c}3 \\
\text { n (\%) }\end{array}$ & \\
\hline \multirow{2}{*}{ S } & Há classificação do motivo de consulta & $147(98,7)$ & $167(98,8)$ & 0,640 & $59(96,7)$ & $192(99,0)$ & $63(100)$ & 0,237 \\
\hline & A anotação é explícita & $71(47,7)$ & $79(46,7)$ & 0,480 & $23(37,7)$ & $102(52,6)$ & $25(47,2)$ & 0,053 \\
\hline \multirow{2}{*}{ O } & As anotações demonstram o estado do paciente & $95(63,8)$ & $115(68,0)$ & 0,246 & $33(54,1)$ & $131(67,5)$ & $46(73,0)$ & 0,067 \\
\hline & As anotações são explícitas e entendíveis & $110(73,8)$ & $144(85,2)$ & 0,008 & $47(77,0)$ & $157(80,9)$ & $50(79,4)$ & 0,800 \\
\hline A & Há classificação de diagnóstico & $145(97,3)$ & $166(98,2)$ & 0,431 & $60(98,4)$ & $189(97,4)$ & $62(98,4)$ & 0,850 \\
\hline \multirow{2}{*}{$P$} & Há classificação de procedimentos & $139(93,3)$ & $168(99,4)$ & 0,003 & $60(98,4)$ & $187(96,4)$ & $60(95,2)$ & 0,627 \\
\hline & Há anotações explicitas de plano e procedimentos & $37(24,8)$ & $36(21,3)$ & 0,270 & $15(24,6)$ & $49(25,3)$ & $9(14,3)$ & 0,188 \\
\hline
\end{tabular}

Nota: Grupo etário: $1:<18$ anos; $2: \geq 18$ e $<65$ anos; $3: \geq 65$ anos.

marcada para o paciente que para o médico. E se este não tiver o cuidado de anotar ao menos os tópicos do que colheu, informou, planejou e aconselhou a um paciente, poder-lhe-á acontecer a confrontação futura, por um colega, por um paciente, ou por si mesmo, sobre o que terá acontecido. ${ }^{11}$

Esse é o fulcro do trabalho presente em que, através de uma lógica meramente quantitativa de verificação da existência de informação em consultas passadas, se procura perceber a frequência da informação que definimos como importante segundo as premissas do método Weed-SOAP.,9

Metodologicamente, as questões éticas de reserva de confidencialidade foram devidamente acauteladas, ao serem os médicos de cada ficheiro a verificar a informação e ao não serem conhecidos os dias de consulta estudados, por as folhas de sorteio terem sido destruídas. As questões de deontologia foram também acauteladas ao serem os próprios a estudar o seu desempenho, assim querendo, com este seu trabalho, aprender e melhorar.

Como viés, podemos considerar o de interpretação do próprio trabalho, ao ser a informação de "A anotação explica classificação", "As anotações demonstram o estado do consulente", "As anotações são explícitas e entendíveis" e "Há anotações que explicitem o plano" criticamente revista por um médico para as consultas efetuadas por vários médicos em cada um dos dois arquivos. Como limitação do estudo, podemos encontrar o fato de a amostra ser de tamanho ligeiramente inferior ao calculado (318 em 332), o que poderemos explicar, apenas, por questão de particularidades dos dias examinados.

Uma pesquisa efetuada no Índex de Revistas Médicas Portuguesas on-line em 20 de outubro de 2012 não mostrou qualquer artigo nesta área quando pesquisamos pelas palavras-chave, informação e/ou classificação e/ou anotação na área da MGF ou noutra área médica.

Assim, os resultados presentes devem ser lidos com a máxima cautela.

A avaliação realizada foi apenas a da existência ou não de informação e não da sua qualidade. Da mesma forma, este trabalho não deve ser lido como de avaliação de qualidade.

Este trabalho deve ser entendido como o início da descoberta de uma "lista de verificação positiva" para que, numa consulta, se realizem, pelo menos e sistematicamente, as atividades condizentes para que no futuro esteja disponível a informação necessária para que o médico, outros médicos e o paciente percebam o que se passou num contato médico-paciente. 
Dado o já longo tempo de trabalho em MGF de alguns dos autores, os presentes pontos de análise foram pensados como os de mais relevante reflexão para cada capítulo do método de coleta e organização da informação.

Partindo da premissa de que todas as consultas devem ter classificação, neste trabalho podemos verificar que a classificação pela ICPC-2 está presente na quase totalidade das consultas efetuadas no ambiente do Sistema de Apoio ao Médico (SAM) (o programa de informática que utilizamos) e utilizando o método de Weed-SOAP nos campos S, A e P. Já as anotações aparecem em menor frequência.

De fato, só há anotações explicativas de classificação em $\mathrm{S}$ em 47,8\% das consultas, sendo este capítulo da maior importância para o conhecimento da agenda dos problemas levados pelo paciente para o contato com o médico. Como explicação para este fenômeno, podemos apenas pensar que os médicos preferem classificar a anotar, devendo para o futuro haver o cuidado de anotar, pois uma classificação nem sempre consegue explicitar bem uma queixa.

Já quanto ao campo O, "As anotações demonstram o estado do consulente" surgem em $66,9 \%$ das consultas que têm classificação em S e "As anotações são explícitas e entendíveis" surgem em 80,9\% das consultas. Estes resultados são interessantes na ótica da explicação do estado do paciente. Os registros da semiologia foram enquadrados na lógica da explicitação e compreensibilidade. Não conseguimos demonstrar as razões pelas quais não estão sistematicamente realizados.

Em P "Há anotações que explicitem o plano" apenas em 36,90\%. Tal parece ser claramente uma insuficiência, pois em MGF muita informação é passada ao paciente, muitas propostas são realizadas e muitos contratos terapêuticos são estabelecidos com alvos definidos a atingir.

Para as diferenças entre gêneros, não podemos encontrar qualquer explicação. Pelos grupos etários considerados e apesar de não haver diferenças significativas, sempre salientamos que é nos mais jovens que há mais anotações quanto ao plano a seguir, o que será eventualmente devido à transmissão de conselhos às mães em consultas de saúde infantil.

É possível que haja excessivo enfoque na classificação perdendo-se eventual qualidade de informação linguística e semiológica sobre o motivo e consulta, sobre avaliação e sobre o plano. ${ }^{11-14}$

\section{Conclusões}

Numa análise e 318 consultas, verifica-se:

Em S, 98,7\% das consultas tiveram classificação e há anotações explicativas de classificação em 47,8\%.

Em O, em $66,0 \%$ das consultas as anotações demonstram o estado do consulente e em $79,9 \%$ as anotações são explícitas e inteligíveis.

Em A, há classificação(ões) que permita(m) pensar em diagnóstico em 97,8\% das consultas;

Em $\mathrm{P}$, há classificação de procedimentos ou plano em $96,5 \%$ das consultas e há anotações que explicitem o plano em $23,0 \%$.

Parece haver campo para uma mais completa coleta da informação na consulta, sendo este trabalho um alerta a todos para a importância de uma boa e adequada mistura de classificação e anotações a realizar em cada consulta em todos os campos do método de Weed-SOAP para que, em plenitude se possa perceber e comparar toda a informação na consulta. 


\section{Referências}

1. Ordem dos Médicos. Código Deontológico [Internet]. Lisboa: OM; c2006-2009 [acesso em 2012 Out 26]. Disponível em: https://www.ordemdosmedicos.pt/?lop=conteudo\&op=9c838d2e45b2ad1094d42f4ef36764f6\&id=cc42acc8ce334185e 0193753adb6cb77 Portuguese

2. Decreto-Lei n.ำ 46/2007 (PT). Diário da República, 1.. a série - N. 163 - 24 de Agosto de 2007 [acesso em 2012 Oct 27]. Disponível em: http://dgarq.gov.pt/files/2008/09/46_2007.pdf no seu artigo Artigo 7.ำ

3. Decreto-Lei n.ำ 12/2005 (PT). Diário da República, 1. a série - N. 18 - 26 de Janeiro de 2005 [acesso em 2012 Out 26]. Disponível em: http://www.cnpd.pt/bin/legis/nacional/Lei12-2005.pdf

4. Ramos V. A consulta em 7 passos. A consulta: pilar central da medicina geral e familiar. Lisboa:VFBM Comunicação; 2008.

5. Caeiro R. Registros clínicos em medicina familiar. Lisboa: Instituto de Clínica Geral da Zona Sul; 1991.

6. Queiroz MJ. SOAP Revisitado. Rev Port Clin Geral. 2009;25(2):221-7.

7. Rodrigues JG. Por que consultam os utentes o seu médico de família? Rev Port Clin Geral 2000;16(4):442-52.

8. Weed LL. Medical records that guide and teach. N Engl J Med. 1968;278(11):593-600. DOI: http://dx.doi.org/10.1056/ NEJM196803142781105

9. Weed LL. Medical records that guide and teach. N Engl J Med. 1968;278(12):652-7. DOI: http://dx.doi.org/10.1056/ NEJM196803212781204

10. Comissão de Classificações da Organização Mundial de Ordens Nacionais, Academias e Associações Académicas de Clínicos Gerais/Médicos de Família. Classificação Internacional de Cuidados Primários. 2a ed. Comissão de Classificações da WONCA; 2011 [acesso em 2012 Oct 26]. Disponível em: http://www.acss.min-saude.pt/Portals/0/apmcg_ICPC\%20 v\%201.7.pdf

11. Braga R. Os registros clínicos e a codificação. Rev Port Med Geral Fam.

2012;28(3):155-6.

12. Pinto D, Corte-Real S. Codificação com a classificação internacional de cuidados primários (ICPC) por internos de medicina geral e familiar. Rev Port Clin Geral Fam. 2010;26(4):370-82.

13. Melo M. O uso da ICPC nos registros clínicos em medicina geral e familiar. Rev Port Med Geral Fam. 2012;28(4):245-6

14. Pinto D. Classificar motivos de consulta e procedimentos com a ICPC na prática clínica? Rev Port Med Geral Fam. 2012;28(4):247-8.

15. Conselho Nacional de Ética para as Ciências da Vida. Parecer sobre informação de saúde e registros informáticos de saúde. Portugal: 2011 [acesso em 2012 Out 15]. Disponível em: http://www.cnecv.pt/admin/files/data/docs/1318269169_ CNECV\%20P_60_2011\%2010.10.11.pdf 